\title{
M2M Device Connectivity Framework
}

\author{
Maman Abdurohman ${ }^{1}$, Aji Gautama Putrada ${ }^{1}$, Sidik Prabowo $^{1}$, \\ Catur W. Wijiutomo ${ }^{1}$ and Asma Elmangoush ${ }^{2}$ \\ ${ }^{1}$ School of Computing (SoC) Telkom University Bandung, INDONESIA \\ ${ }^{2}$ Fraunhofer FOKUS Institute, GERMANY \\ \{abdurohman, ajigps, pakwowo, caturwijiutomo\}@telkomuniversity.ac.id, \\ asma.elmangoush@alumni.tu-berlin.de
}

\begin{abstract}
This paper proposes machine-to-machine device connectivity (M2M-DC) framework to enhance efficiency of smart metering system by controlling electrical devices in a building. Internet of Things (IoT) is a trend technology implemented in many areas, of which the utilization to enhance the efficiency of power consumption is significantly important. There are several smart metering solutions have been developed in recent years, however, they are silo solutions that pay a little attention to scalability. To ensure scalability, a framework that rules number of sensors, actuator and devices are required. M2M-DC is a proposed framework that connects the nodes with sensors and actuators to the backend system based on the rule set. Some experiments are performed to measure the effectiveness of the system. The results show that the M2M-DC framework can control energy consumption, achieving higher energy efficiency of $26.35 \%$, regarding the rule set. Based on the Wilcoxon method, the significance of the increase of efficiency on power consumption is confirmed. The scalability of proposed framework is also verified by varying sensors, actuators and devices.
\end{abstract}

Index Terms: Internet of Things (IoT), M2M, Smart Metering and Framework

\section{Introduction}

Internet of things (IoT) has become a technology that is very prominent in the last 3-4 years. It is not only a topic of conversation in the technology area, but has also become the focus in IT business. Along with the development of services that run over Internet technology, IoT also pushes up the growth in the use of the devices that are connected to the internet. Since 2015 the number of online devices has been reaching 6.4 billion around the world. It is predicted to continue to grow by $30 \%$, that is 5.5 billions devices in just one year (2016) [1].

One important part of IoT is the Machine-to-Machine (M2M) technology. M2M is a communication type of form that involves several components of the entity (device / Machine) to work together, to minimize or even eliminate the need for human interaction (automatic). Further growth of M2M technology, allowing it to facilitate the interconnection between devices, is not limited to the massive computing resources device, but will be able to accommodate communication between devices with minimal resource as well. Some examples of machine type devices are sensors, mote, and actuator.

ETSI referenced M2M architecture is one of standard of M2M. This standard consists of two parts i.e. Networking and Device \& Gateway domains. Device \& gateway domain connects the system to M2M device through M2M gateway. M2M service capability layer is part of networking domain that connect between gateway and user application [2].

M2M system requires numerous components that communicate with each other. This motivates the development of many platforms to accommodate the entire components building M2M communication that complies with particular standard. This platform is obviously accelerating the development of M2M as well as IoT. One of M2M platform that complies with the ETSI and OneM2M standard is the OpenMTC. This system consists of network platform

Received: April $3^{\text {rd }}, 2017$. Accepted: September $23^{\text {rd }}, 2017$

DOI: $10.15676 /$ ijeei.2017.9.3.2 
and gateway platform. Network platform serves user by providing network, data, and device API [3]. There are several applications have been developed based on this platform i.e. [4] and [5].

The rapid growth of the world population, especially in Indonesia, leads to an increase in the need for both housing as well as office space, which will have an impact on the total energy required. The problem of controlling electrical power usage in both housing and office sectors are important to be solved.

The utilization of IoT supporting the better efficiency of power consumption is urgently needed. There are several smart metering solutions that have been developed to address this problem as in [6] [7] and [8]. However, these solutions are silo solutions that pay little attention to scalability. Meanwhile, the problem of energy efficiency of electric energy requires a widescale solution, which is also requiring a framework to increase efficiency that supports wider usage scales.

The main contribution of this paper is the proposed M2M-DC framework as the many-tomany communication model that connects number of applications with nodes. These nodes are connected to number of sensors, actuators, and devices. The use of M2M middleware is the key that enables the framework to handle a wide range of sensors, actuators and devices.

\section{Related Work}

Based on the National Intelligence Council definition, the Internet of things (IoT) refer to the general idea particularly everyday objects that can be read, recognized, be located, has an address and can be controlled via the Internet [1].

In this case, the everyday objects are including:

- Electronic devices that are often used everyday

- High technology Products such as car

- Objects other than electronic

The ability of everyday objects to start to connect to the internet as the result of VLSI and SoC technology, is not only allowing the capability and high process speeds, as seen on PC, but also allows small applications to run on small systems. There is a growing need for small embedded processor such as the microcontroller 8,16 or 32 bits with the integration of the network interface on the SoC. A device with that type of processor, and a small one, enables energy saving device but would be able to run TCP / IP stack in it.

Regarding the complexity of the above everyday objects, most of IoT solutionsare made in ad-hoc framework, relying heavily on the business domain and platform,so that the implementation of IoT for a very different cases and architectures that do not fit together implemented in the field. Programming on IoT environment is not just abouta TCP / IP socket connection but how to connect the components of the device to the cloud domain. Sincethere are so many optionsand a large scope, so that the implementation of a particular standard and utilisation of framework will help tosimplify the IoT implementation.

One of standards used is a communication protocol, usually the IoT framework, is built on communication protocols such as TCP/IP. It is also possible to use higher layer protocol at the transport layer such that MQTT, AMQP or XMPP.In addition to the communication protocol, the next challenge lies in the scope of application of IoT itself. One consideration is the number of nodes; numbers of solutions designed for millions of nodes.

One other approach to simplify the IoT solution by using the Architecture Reference Model which is the result of the IoT- A European research project [9]. This model describes the conceptual entities and relation between object. ARM is a useful tool to allow stakeholders a common language between M2M and IoT.

Several reference models and reference architectures have been created; a popular one is the ETSI M2M architecture [2]. ETSI standard separates between Network domain as well as device and gateway domain. In 2009 ETSI set up a technical Committee which focuses to 
produce communication standard between machines[10]. Presently, the Telecommunications sector of the ITU-T has been active in setting the standard IoT since 2005[9]. In 2013, ITU-T made recommendations that describe overview of the IoT reference and functional model [2]. ITU-T standard consists of four layers i.e. Application, service \& application support, network, and device layers. Next, the IETF (Internet Engineering Task Force Architecture Fragments) has defined three groups that deal with M2M and IoT [13]. These groups are 6LoWPAN [10], Constrained Restful Environment (CoRE) [11], and ROLP and Lossy Network [12].

Framework Implementation is another way to simplify IoT solution, by formulating some rules to connect the nodes of the device domain to application domain. Some IoT frameworks provided by vendors include: Eclipse IoT Projects [14], AllJoyn [15], IoTivity [16] and Kaa [17].

In many cases a vendor rarely builds its own IoT solution of the framework from the beginning, due to the complexity and components variabilityconnected from the microcontroller application in the device, the middleware through the utilized database server.

Smart energy in buildings is an important research topic, since eficiency in energy usage is important for the sustainability of human life. Some researches propose the utilisation of a framework for energy management requirements in the smart building implementation. One of studies in buildings smart energy is to implement a framework to achieve three aspects. These include: energy usage monitoring, capitalization and energy usage evaluation and IoT system for controlling [18]. The use of multi-agent which coordinates each other to manage energy [19] is one way to obtain energy information from the nodes that undertake monitoring energy. In addition, framework to applying the rules of energy efficiency is also requires the rules sets implementation which is controlling the usage threshold by considering the patterns of energy usage e.g. by occupancy [20]. Hence, the implementation of smart energy building can be more efficient.

There are many solutions of smart metering such as in [6]. In this research, the author maximizes the strength of IoT to optimize energy consumption efficiency, where users are provided with ease by its metering display and energy limitation by manual threshold setting. The weakness of this system that it still uses a Silo-based framework. Another approach as in [7] is about smart metering where there are many smart metering nodes and each node is connected to a mesh topology network. The network utilizes FHSS (Frequency Hop Spread Spectrum). Although being mesh but each node still represents a Silo-based framework. The paper also doesn't provide metrics that shows the advantage of such mesh topology network. Also, another solution as in [8] is about multi node smart metering system. The system utilizes Power Line Communication (PLC) for connectivity inter each device. Although scalable in macro aspect this paper lacks contribution in controlling, where each device only provides information for user awareness through what is called IHD (In-Home Display). The research does prove to reduce energy consumption, not by its horizontal based architecture, but emphasizes more on monitoring and display.

\section{Proposed Framework: M2M-DC}

IoT development framework in general comprises of three main parts, namely: application, middleware and device. The third part of this topology can be divided into two parts: the server and the node. Viewed from back end system to front end system there is a Many-to-Many (M$\mathrm{N})$ relationship. In the context of many-to-many relationship there are number of IoT applications that work independently from one another on one hand, and a number of devices that connected to the device enabler, on the other hand. Many-to-many mapping shows that there is one device that can be accessed by one or more of IoT application and also, there is one IoT application can access one or more devices, as shown by Figure 1. 


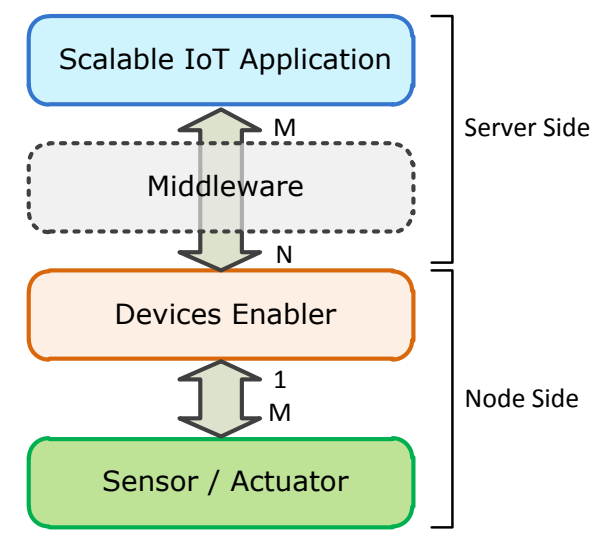

Figure 1. Proposed M2M Device Connectivity Framework.

Middleware itself comprises of several parts. On the top, there is the IoT API connected with the IoT application. The connection between the IoT application and middleware is a many-to-many. An IoT API can be used by one or several IoT applications and one IoT application can use one or several IoT API contained in the middleware. The IoT API provides various services given by the middleware that is connected to number of devices. Figure 2 . shows the schematic of a middleware that connects to an IoT Application and with devices at once.

On the bottom, there is a gateway device that is connected to the node side with many-tomany type of connectivity mapping. Node side comprises of two parts: a devices enabler and a set of sensors/actuators. In the device enabler, there are two important components namely master and slave. The function of the former is to control the slave, while the slave is directly connected to devices in the form of sensors and actuators as shown in Figure 3. The master functions are connected to the slave as well as to the server side through a wireless or wire line network.

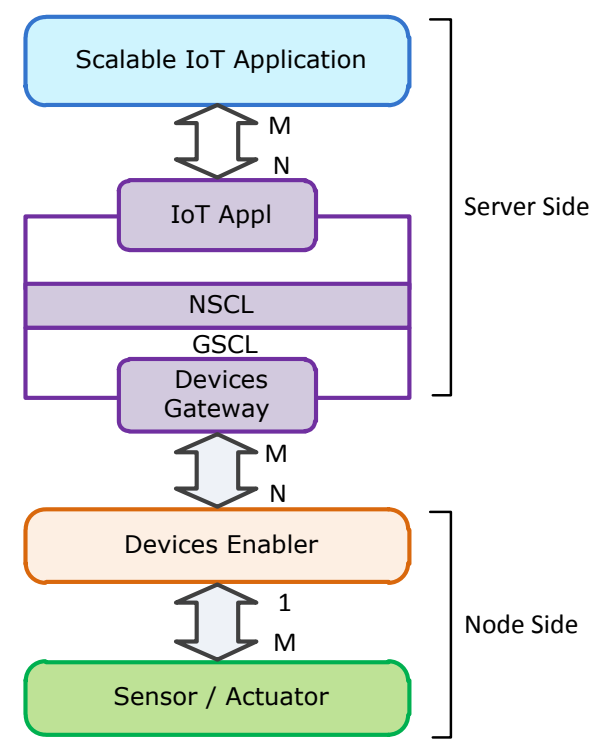

Figure 2. Server Middleware Architecture. 


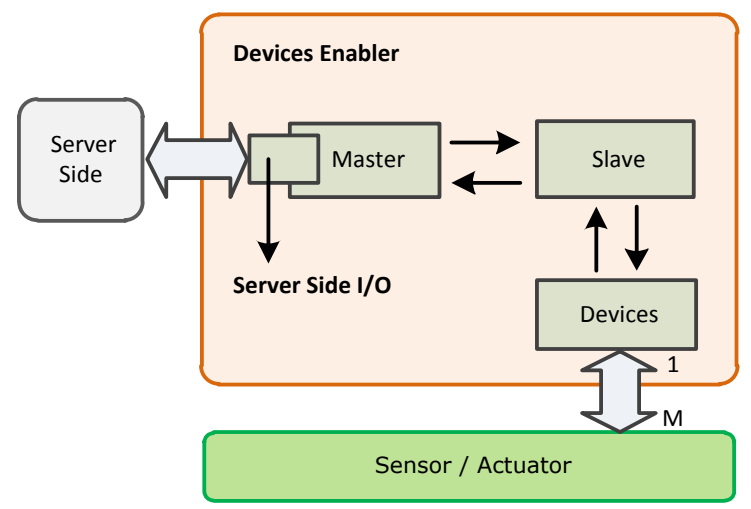

Figure 3. Devices Enabler Architecture.

\section{Smart Metering System}

M2M-DC framework that is proposed in this paper is implemented on the smart metering environment case study. The discussion in this part of the paper, is a general explanation of the smart metering to its testing and the analysis.

In a horizontal IoT platform framework, scalability is a major concern. Horizontal platform is made so the performance of a system, for example smart metering, is not disrupted when its scale is enlarged. Testing with the case study of smart metering is intended to see the performance of this system when the scale is enlarged. That is why in this test three electrical outlet channels are used in one smart metering end node.

\section{A. The Smart Metering Environment}

The purpose of the Smart Metering System implementation is to improve efficiency in electrical energy consumption of a system. Data from Environmental international Agency (EIA) show that in 2010, more than $70 \%$ in china and $63 \%$ in United Stated electricity comes from fossil fuel combustion, with most developed countries within the same range [21]. Form this statistic then estimated that earth has only few decades of supply of the Non-renewable sources. These are the main reason why the research to reduce the energy consumption has become interesting. To achieve these objectives, the Smart Metering System has two functions, namely the function of monitoring and controlling.

Monitoring function of the system is displaying energy consumption of the system environment. Energy consumption shown is the total energy consumption and the channel energy consumption, one channel will be connected to one electronic device. The workings process of the monitoring function is, the system performs sensing of the voltage and current from the system, the voltage and current are then processed into electric power consumption, finally energy power consumption is displayed in the graphical form of power consumption change against time.

The controlling function of this system is to the use of electrical equipment. The systems store an electrical energy threshold, of which the system would try to keep the electricity consumption in periods of time to not exceed that threshold. The controlling function works as, first the system calculates the current energy consumption based on the graphic of power consumption versus time, then the system performs computation based on the three aspects, namely the energy consumption threshold, the current energy consumption, and some rules. The results of this computation may result in blockage of some electrical equipment use in the system. The system would comprise of two parts, these are the M2M server run by OpenMTC Server and the M2M device or End System. The End System is responsible for sensing and actuating. Figure 4. shows the entire smart metering system. Meanwhile, Figure 5. is a block diagram that explains how the End System works; 


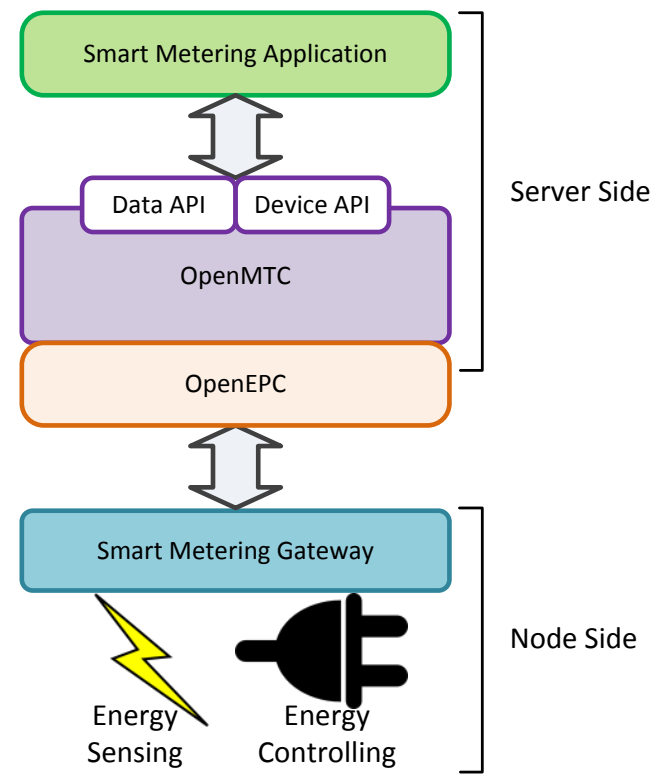

Figure 4. Smart Metering System.

End System will consist of two sides, the Master and Slave sides. The Master side is the side that serves the communication with OpenMTC Server; this side will consist of Wi-Fi communication module. The Slave side is the side that does actuating and sensing; this side will consist of a microcontroller system that is connected to sensors and actuators.

\section{B. Hardware and Software Specifications}

The following is the Smart Metering End System hardware specifications:

- ESP-12 for wireless communication, as well as Master Microcontroller

- Arduino Nano as Slave Microcontroller

- Relay as actuators or contact breakers

- Split Core Current Transformer type SCT013-00 as the total current meter

- Current Transformer type through hole ZMCT103 as the channel current meter

- Voltage Transformer as the voltage meter

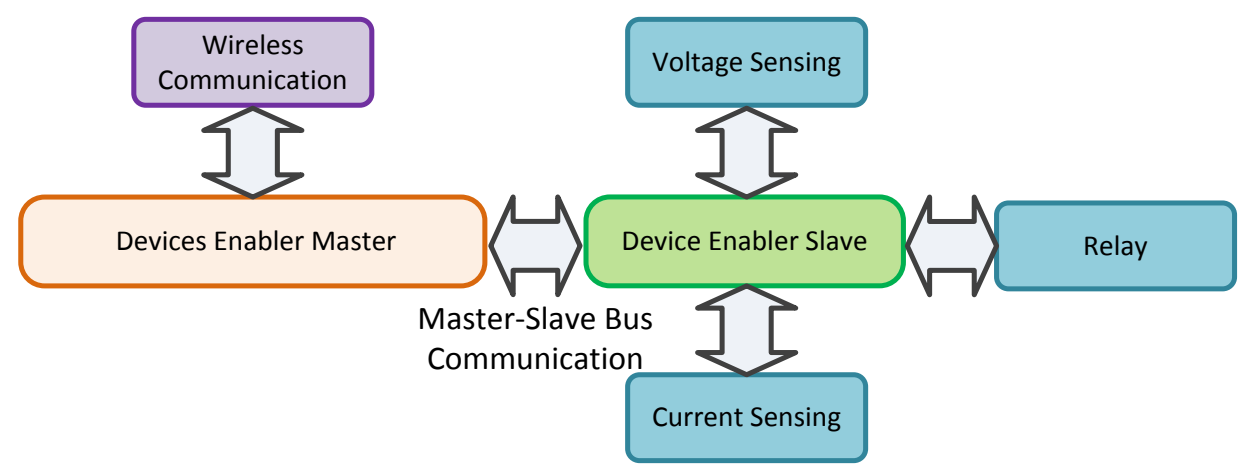

Figure 5. Device Enabler Block Diagram. 
The ESP-12 as the Master Device and Arduino Nano as the Slave Device will communicate via $\mathrm{I} 2 \mathrm{C}$ interface.

Figure 6. contains a Data Flow Diagram (DFD) that explains the process that occurs in the IoT Server. IoT Server is responsible for data storage and Rules calculation. Communication with data enabler and communication with application request are kept asynchronous, so that consumer producer concept can occur.

To operate the slave and master, a software is written on top of each of them. For example, the master, has been given the ability to communicate data to The OpenMTC Server. The master will command the slave, through communications (I2C) to sense voltage and current, and send them simultaneously to the server.

In the other way around, communication from Server to Slave happens when a control is sent. Control is in form of switching a power channel. The Master will receive this order and pass it to the slave via Bus communication. A data flow diagram explaining the process of the enabler master is provided by Figure 7.

The slave will wait for a request from the master, when given, it sends acquired voltage and current data, and such data is received from three different channels. After doing sending operation the slave will wait for a controlling request from the master. If present, the slave will operate the controlling request by switching the relay of the channel desired by the request sent.

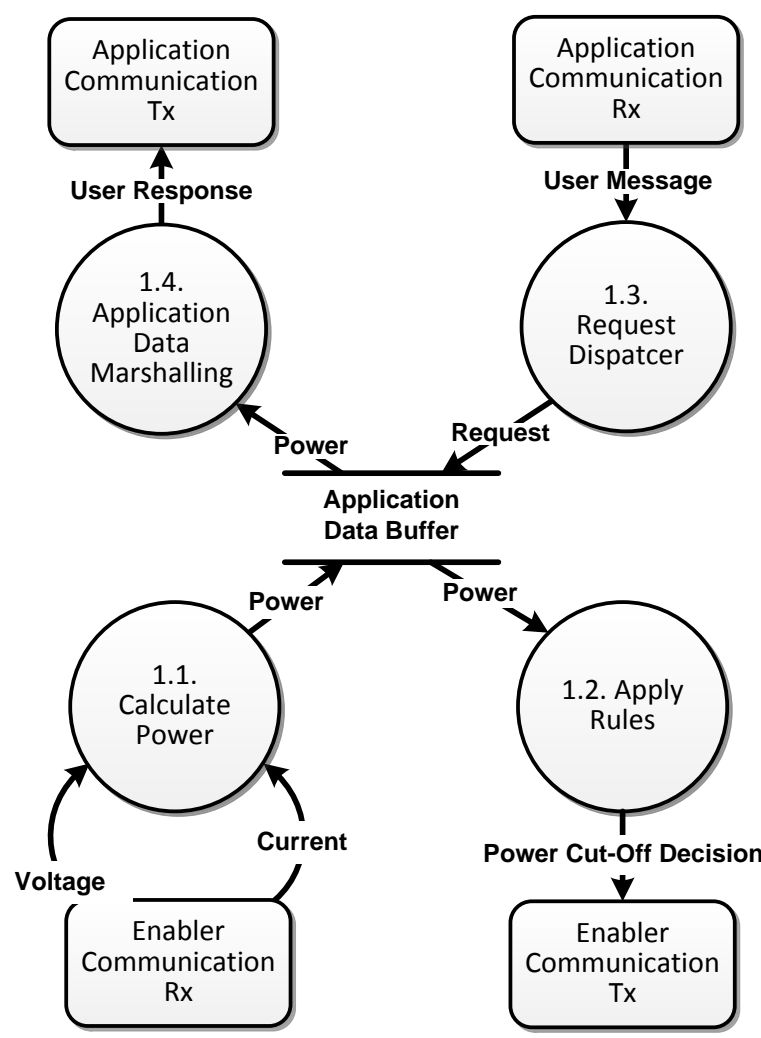

Figure 6. Data Flow Diagram of the IoT Server. 


\section{Two categories of Smart Metering System Rules}

1. Scheduling Rule

The Channel with this Rule will turn on and turn off on a set schedule

2. Energy Threshold Rule

The Channel with this Rule will turn off after a set energy level

\section{Notes:}

1. There are three provided channels

2. Each channel can be assigned by any Rule category

3. Energy Threshold is in Wh

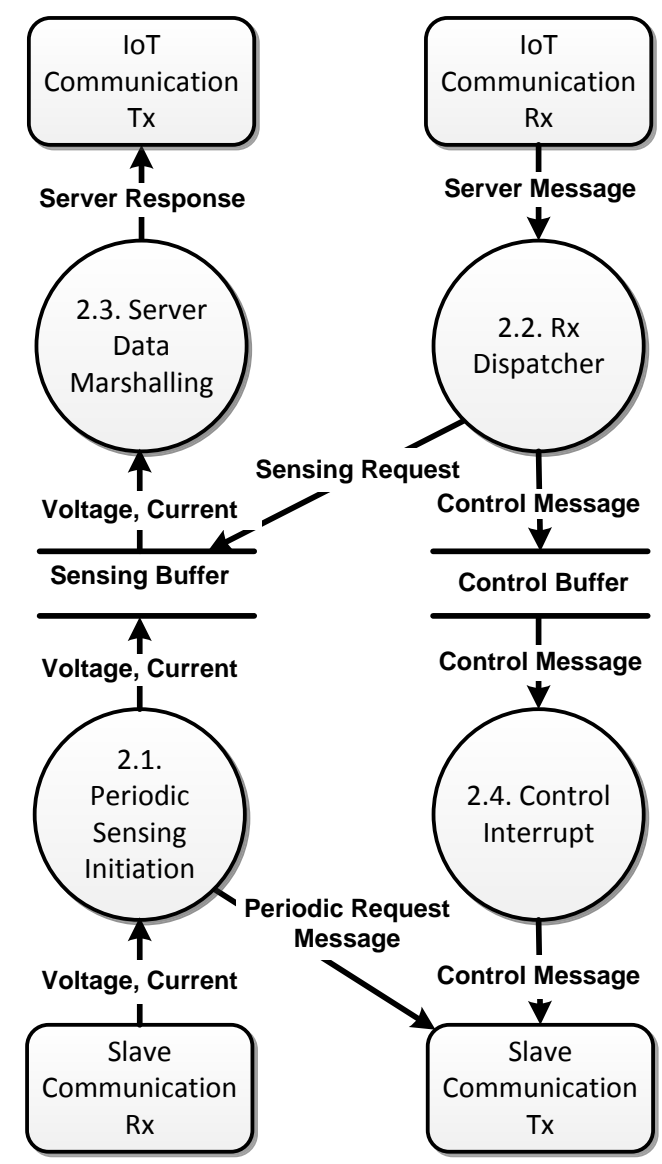

Figure 7. Data Flow Diagram of the Enabler Master.

In this system, there would be three channels of electrical devices. Additionally, the user manually determines which rule will be assigned to which channel. The Rules are defined as previous. The whole smart metering system flow chart diagram is showed in Figure 8. 


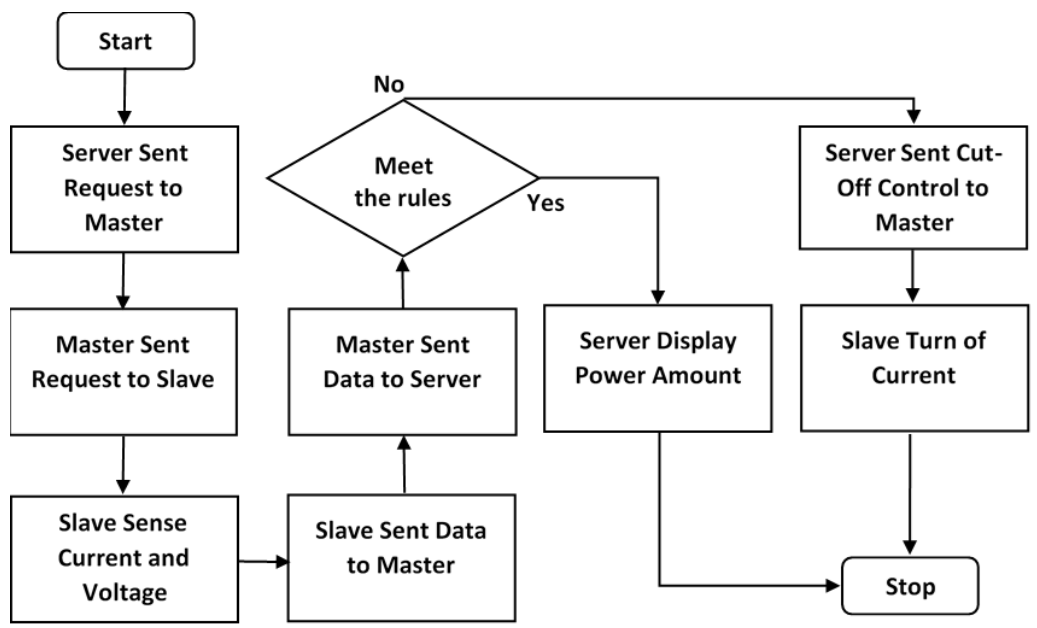

Figure 8. Flow Chart of Smart Metering System.

\section{Results and Discussion}

The main scenario of Smart Metering System test is comparing the electricity energy of a monitored test bed without rules and with rules. The rules applied are the Smart Metering System Rules defined in the previous chapter. Two results are hoped to be obtained by the test; namely Energy Efficiency and Significance. Energy Efficiency is derived from comparing Energy Consumption of each test and Significance is derived from Wilcoxon Method [22]. The environment of the test, or the test bed, is as shown in Figure 9.

The test bed is equipped with three different channels. Each channel is connected to different electrical appliance.

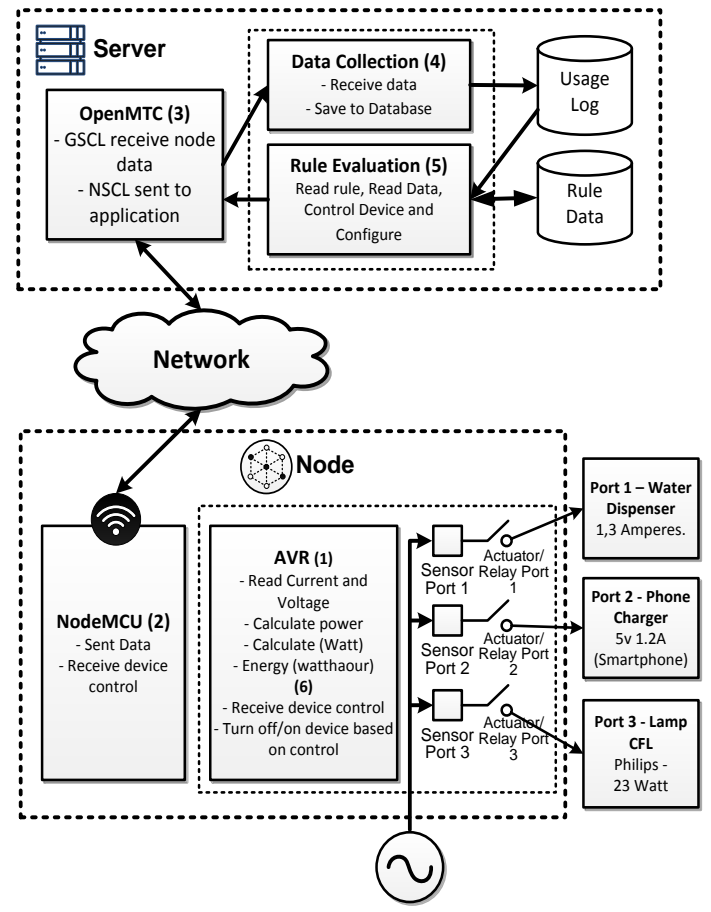

Figure 9. Testbed environment. 
The following is the explanation of each electrical appliance connected:

1. Channel 1 is connected to a 1,3A Water Dispenser with Heating Capabilities

2. Channel 2 is connected to a $5 \mathrm{v}, 1,2 \mathrm{~A}$ smart phone charger

3. Channel 3 is connected to a 23 Watt CFL light.

There are two types of Smart Metering Rules defined in the previous chapter, mentioned, scheduling rule and energy threshold rule.

Each channel mentioned above is given one of the two rules. The description is as follow:

1. Channel 1 is given the scheduling rule

- The off schedule of Channel 3 is 01:30 a.m. to 04:00 a.m. UTC

2. Channel 2 is given the scheduling rule

- The on schedule of Channel 2 is 01.00 a.m. to 06.00 a.m. UTC.

3. Channel 3 is given the energy threshold rule

- The energy threshold of Channel 3 is 120 Watthour per Day.

There are 2832 data for each Test. The data contains voltage, current, and Wattage, each sampled every 30 seconds. We can conclude that the test is undertaken for 84.960 seconds or 23 hours and 36 minutes.

\section{A. Power Characteristics Observation}

We will call the test without Rules Test 1 and the test with Rules Test 2 . The results can be seen in the graphic of Figure 10.

From Figure 10. (a) we can observer the pattern of each appliance. Channel 1 with water dispenser has increase and decrease characteristics. This is due to the character of the appliance that will heat the water when reaching certain heat temperature.

Channel 2 with a phone charger has low but stable power consumption. This is understood because a smart phone charger has low voltage output (usually 5 Volts). Channel 3 with an energy saving light bulb has similar characteristics to a phone charger, low and stable, but is considerably higher than Channel 2, that is around 22 Watts. This is appropriate to the specification of the CFL Light that is 23 Watts.

The Power Consumption of each channel in Test 2 is show by Figure 10. (b). It can be seen in Test 2 that the Power Consumption of Channel 1 becomes zero near the end of the Test. This is due to the schedule rule applied to the Channel.

The Power Consumption of Channel 2 becomes Zero because time schedule for Channel 2 is on for 5 hours. It is similar with Channel 2, Channel 3 also becomes zero but because the energy consumption threshold has been reached. In documented test results, Channel 3 becomes zero after 154 minutes. It turned off after the Energy consumption reached 120,88 Watthours, appropriate to the rules applied.

\section{B. Energy Consumption}

The comparison of energy consumption of Test 1 and Test 2 is shown in Figure 11. From Test 1, total Energy Consumption results for Channel 1, Channel 2, and Channel 3 respectively are 1478,37 Watthours 6,01 Watthours and 523,05 Watthours. From all three results, it can be seen that Channel 1 is the highest. This is understandable considering a Water Dispenser with heating capabilities has bigger power consumption than a smart phone charger and an energy saving light bulb. Cumulative Total Energy Consumption for Test 1 is 2007.42 Watt hours.

From Test 2, total Energy Consumption results for Channel 1, Channel 2, and Channel 3 respectively are 1410,71 Watthours, 2,63

Watthours and 120,90 Watthours. Cumulative Total Energy Consumption for Test 2 is 1478.37 Watt hours. 


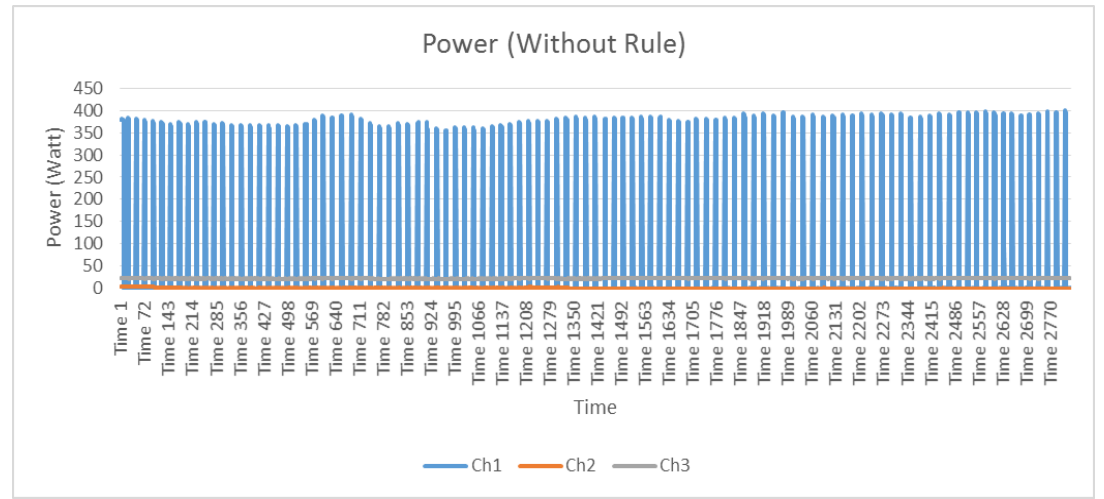

(a)

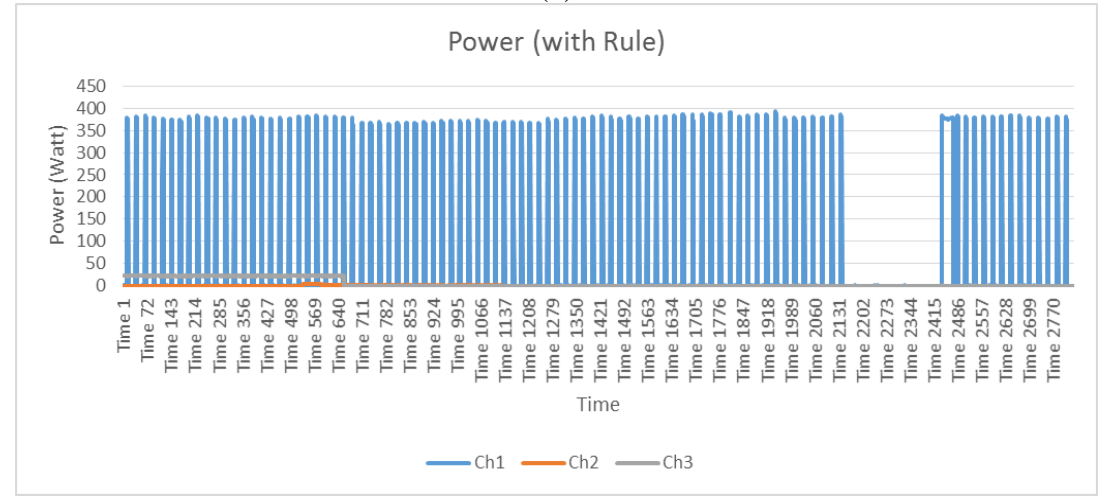

(b)

Figure 10. Measurement Results Graphic Comparison of Proposed Smart Metering System. (a) Rules are not applied and (b) Rules are applied.

Energy Consumption of each channel of Test 2 are lower than Energy Consumption of each channel of Test 1 . It can be concluded that Smart Metering Rules applied to each channel provides energy efficiency. Energy of each channel of Test 2 respectively is more efficient $95.42 \%, 43.83 \%$, and $23.11 \%$ than Test 1 .

Total Energy Consumption of test scenario 1 (without rules) is 2007.42 Watt hours meanwhile total energy consumption of test scenario 2 (with rules) is 1478.37 watt hours.

Efficiency $=(2007.42-1478.37) / 2007.42 \times 100 \%=26.35 \%$.

It shows that the efficiency of decreasing energy consumption is about $26.35 \%$.

\section{Significance Test}

Wilcoxon Method is used to discover the significance of the Smart Metering System Rules to Energy Consumption[18]. In this Method, 2832 data of energy consumption as shown in Figure 11. that are obtained each from Test 1 and Test 2 are compared.

The hypotheses are as follow:

- Ho: Median (Difference) $=0$, or the Smart Metering System Rules does not give significance to energy consumption in the environment

- Ha: Median (Difference) $\neq 0$, or the Smart Metering System Rules does give significance to energy consumption in the environment 


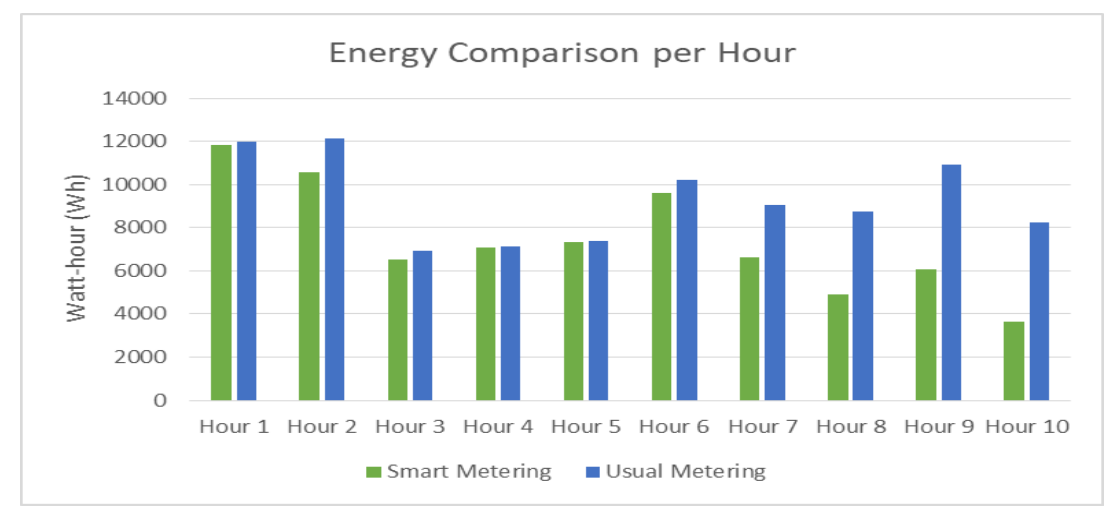

Figure 11. Comparison of power consumption with and without Smart Metering

The significance level $(\alpha)=0.1$, being the biggest significance level value. Meaning that we are searching for big significance in the effect of the application of Smart Metering System Rules.

After putting the two samples together, organizing it in ascending order, adding ranks to the order, the following equation is applied:

$$
z=\frac{R-\frac{n_{1}\left(n_{1}+n_{2}+1\right)}{2}}{\sqrt{\frac{n_{1} n_{2}\left(n_{1}+n_{2}+1\right)}{12}}}
$$

The result obtained from that formula is -130.365 . From that result, since $|z|=130.365>z^{*}$ $=1.64$ it is concluded that the Null hypothesis $\mathrm{H}_{0}$ is rejected. Meaning that the Smart Metering System Rules does give significance to energy consumption in the testbed environment.

\section{Conclusion}

In this paper, we have proposed M2M-DC framework as a communication framework to support smart metering systems accommodating scalability. This framework is a horizontal platform system that defines the entire concept of connectivity of M2M devices to applications. The Framework describes the complete communication from end-node to server side and the definition of the end-node system. In the complete system, a mapping relationship between endnodes and applications was defined, while the detailed device enabler framework was structured as a general pattern of an end-node to access sensor data and to control devices. Based on the experiments, we found that energy consumption in M2M-DC environment can be increased to $26.35 \%$, which is more efficient compared to conventional system. Based on the Wilcoxon method, the significance of reduction on power consumption is confirmed. The scalability of proposed framework is also verified by varying sensors, actuators and devices.

\section{Acknowledgment}

The authors would like to thank the Research and Community Service (PPM) bureau of Telkom University that has funded the research in international research scheme with the Decree No. 130 / PNLT3 / PPM / 2015. Also, would like to thank the colleagues from PT. Telkom Digital Service Division and Fraunhofer FOKUS Germany. Thanks also to the support team, Pak Anton, Andra, Musa, Fajar, Arigi and Dede. 


\section{References}

[1]. Gartner. "Gartner Says 6.4 Billion Connected 'Things' Will Be in Use in 2016, Up 30 Percent From 2015.” [Online]. Available: http://www.gartner.com/newsroom/id/3165317. [Accessed: 17-Nov-2016].

[2]. T. ETSI, "102 690:" Machine-to-Machine communications (M2M)," Funct. Archit., 2011.

[3]. M. Abdurohman, A. Herutomo, V. Suryani, A. Elmangoush, and T. Magedanz, "Mobile tracking system using OpenMTC platform based on event driven method," in ResearchGate, 2013, pp. 856-860.

[4]. V. Suryani, A. Rizal, A. Herutomo, M. Abdurohman, T. Magedanz and A. Elmangoush, "Electrocardiagram monitoring on OpenMTC platform," 38th Annual IEEE Conference on Local Computer Networks - Workshops, Sydney, NSW, 2013, pp. 843-847.

[5]. A. Herutomo, M. Abdurohman, N. A. Suwastika, S. Prabowo and C. W. Wijiutomo, "Forest fire detection system reliability test using wireless sensor network and OpenMTC communication platform," $20153 \mathrm{rd}$ International Conference on Information and Communication Technology (ICoICT), Nusa Dua, 2015, pp. 87-91.

[6]. A. Geetha and K. Jamuna, "Smart metering system," 2013 International Conference on Information Communication and Embedded Systems (ICICES), Chennai, 2013, pp. 10471051.

[7]. Antonio Liotta, Daniël Geelen, Gert van Kempen, Frans van Hoogstraten, (2012) "A survey on networks for smart-metering systems", International Journal of Pervasive Computing and Communications, Vol. 8 Issue: 1, pp.23-52

[8]. T. S. Choi, K. R. Ko, S. C. Park, Y. S. Jang, Y. T. Yoon and S. K. Im, "Analysis of energy savings using smart metering system and IHD (in-home display)," 2009 Transmission \& Distribution Conference \& Exposition: Asia and Pacific, Seoul, 2009, pp. 1-4.

[9]. M. Bauer et al., "Iot reference model," in Enabling Things to Talk, Springer, 2013, pp. $113-162$.

[10].H. Zhu, Z. Pang, B. Xie, and G. Bag, "IETF IoT based wireless communication for latency-sensitive use cases in building automation," in Industrial Electronics (ISIE), 2016 IEEE 25th International Symposium on, 2016, pp. 1168-1173.

[11].Z. Shelby, "Constrained RESTful environments (CoRE) link format," 2012.

[12]. A. Oliveira and T. Vazão, "Low-power and lossy networks under mobility: A survey," Comput. Netw., 2016.

[13]. "M2M and IOT - A Survey of various Standards | Raja Moorthy | LinkedIn.” [Online]. Available: https://www.linkedin.com/pulse/m2m-iot-survey-various-standards-raja. [Accessed: 25-Nov-2016]

[14]. Al-Fuqaha, A. Khreishah, M. Guizani, A. Rayes, and M. Mohammadi, "Toward better horizontal integration among IoT services," IEEE Commun. Mag., vol. 53, no. 9, pp. 7279, 2015.

[15]. A. Alliance, Introduction to the AllJoyn Framework. 2014.

[16].J.-G. Yoo et al., "A Study of Visual Programming Tools for the Open Source Project IoTivity," Int. Inf. Inst. Tokyo Inf., vol. 19, no. 6B, p. 2369, 2016.

[17]. T. Malche and P. Maheshwary, "Harnessing the Internet of Things (IoT): A Review," Int. J., vol. 5, no. 8, 2015

[18].P. H. Shaikh, N. B. M. Nor, P. Nallagownden, I. Elamvazuthi, and T. Ibrahim, "A review on optimized control systems for building energy and comfort management of smart sustainable buildings," Renew. Sustain. Energy Rev., vol. 34, pp. 409-429, 2014.

[19]. A.L. Dimeas and N. D. Hatziargyriou, "Operation of a multiagent system for microgrid control," IEEE Trans. Power Syst., vol. 20, no. 3, pp. 1447-1455, 2005.

[20]. N. Noury, P. Barralon, G. Virone, P. Boissy, M. Hamel, and P. Rumeau, "A smart sensor based on rules and its evaluation in daily routines," in Engineering in medicine and biology society, 2003. Proceedings of the 25th annual international conference of the IEEE, 2003, vol. 4, pp. 3286-3289. 
[21].X. Yu, C. Cecati, T. Dillon, M. G. Simoes, "New frontier of Smart Grids", IEEE Industrial Electronics Magazine, vol 5, no. 3, pp 49-63, 2011

[22]. R. H. Randles, "Wilcoxon signed rank test," Encycl. Stat. Sci., 1988.

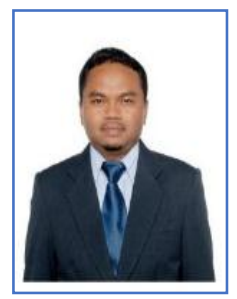

Maman Abdurohman, He has a master degree and a doctoral degree, both obtained from ITB, 2004 for his master, and 2010 for his doctor. He is a lecturer at Telkom University (formerly known as STT Telkom). He has been teaching and researching there since year 2000. His paper has been published in many internationally-referred journals (IJEEI - International Journal of Electrical and Informatics Engineering, Journal of Mechanics and Applied Materials International Journal, International Journal of Information and Education Technology, and more). He has received research grants nationally and internationally from Telkom University and the Ministry of Higher Education from the last three years. Currently he is focusing on certain research areas, namely internet and smartcard technology.

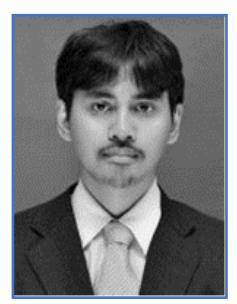

Aji Gautama Putrada, $\mathrm{He}$ is a bachelor and master graduate of Electrical Engineering Department, ITB, both achieved in 2008 and 2013 respectively. His early research was in the area of Broadband Wireless Access (BWA). WiMAX was the subject of his bachelor project, he focused on the same area from 2007 to 2010. After WiMAX, he continued his research to Long Term Evolution (LTE) and completed his Master with this research as his Thesis. The researches currently under progress are in the area of Internet of Things, Embedded Systems, Real Time Operating Systems, Machine Learning, Network Security and Performances, FPGA design, and Smart Cards.

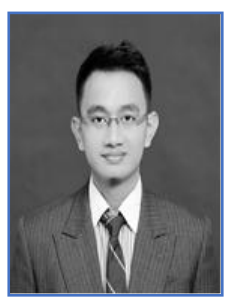

Sidik Prabowo, He is a bachelor and master graduate of School of Computing, Telkom University, both achieved in 2011 and 2014 respectively. He continued his career at Telkom University as a fulltime lecturer and a junior researcher. $\mathrm{He}$ is also involved in national and international research grants from Telkom University and the Ministry of Higher Education of Indonesia. He is a part of the Indonesian Smart Card Consortium. The Consortium has been active for the last two years. He is currently involved in Internet of Things and Machine to Machine Communication researches.

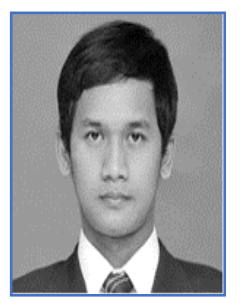

Catur Wirawan Wijiutomo, He obtained his Bachelor degree in Informatics Engineering ITB and is a part of the class of 2005. He received his Master degree from Computer Science, ITB. He is currently continuing his study as a Doctoral Student in the School of Electrical Engineering and Informatics at ITB and also a lecturer with some researches at the School of Computing, Telkom University. $\mathrm{He}$ is involved in researches around Internet of Things, Avionics and Distributed Systems.

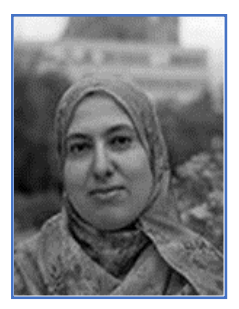

Asma Elmangoush, She has a B.E. and an MS.c degree in computer engineering that was obtained from the College of Industrial Technologies-Misurata, Libya. She also has a Ph.D. degree that was obtained from the Technical University Berlin in 2016. She gained the responsibility to teach a number of courses (for example C++ Programming and Computer Networks) during her time at TUB, being an Assistant Lecturer since 2007. She conducts research in the area of IoT platform and Quality of Service. She was a team personnel for the Misurata Free Zone (MFZ) Company. Asma is currently a lecturer at the College of Industrial Technologies-Misurata, Libya. 